\title{
An evaluation of link estimation algorithms for RPL in dynamic wireless sensor networks
}

\author{
Peter Ruckebusch ${ }^{1}$, Jens Devloo, David Carels, Eli De Poorter ${ }^{1}$, and Ingrid \\ Moerman $^{1}$ \\ Ghent University iMinds, Department of Information Technology (INTEC), \\ Gaston Crommenlaan 8 Bus 201, 9050 Ghent, Belgium, \\ peter.ruckebusch@intec.ugent.be
}

\begin{abstract}
Link estimators are extremely important in dynamic wireless sensor networks for obtaining a good network performance because they drive the decisions made by the routing protocol. Many estimators exist but the quality of their estimation depends on the scenario at hand. In this paper, the impact of the estimator on the network performance is investigated in different networking scenarios. Also the influence of the underlying MAC protocol was evaluated. The evaluation was performed both in simulation and on a real-life testbed.

The results clearly show that there is no link estimator that is best in all scenario's. Another major finding indicates that the results obtained in simulator differ heavily from the testbed results. This illustrates that research findings cannot be solely based on simulation results but also requires real-world experiments. The influence off the underlying MAC protocol is limited on the choice of estimator.
\end{abstract}

Key words: WSN, RPL, dynamic wireless sensor networks, routing, IoT, mobility

\section{Introduction}

Link estimation is extremely important in dynamic Wireless Sensor Networks (WSN) since it drives the decisions made by the routing protocol. Selecting the best path towards the destination is crucial for increasing the overall network performance in terms of throughput, latency and reliability. Moreover, selecting the best links reduces the number of retransmissions and, consequently, the energy consumption.

For this reason, many link estimators for WSNs have been proposed [3]. While complex algorithms, taking asymmetrical links into account, perform better compared to simple estimators, they cause a higher overhead [10]. More intelligent algorithms, such as Four-bit [7], seem to obtain higher Packet Delivery Ratio (PDR) in networks with a high interference level [9]. In dense networks, however, these algorithms can encounter some scalability issues [5], due to variations in different sources of information causing constantly changing metrics [10]. 
Given the numerous dynamic networking scenarios and applications envisaged for WSNs in the Internet-of-Things (IOT), it is to be expected that a single link estimator will not be able to provide the best estimation in all situations. Therefore it is important to know which link estimation algorithms delivers the best results in various scenario's.

To investigate this, several well-known link estimators were implemented for the Routing Protocol for Low-Power and Lossy Networks (RPL) and evaluated both in a simulator and on a real-life testbed, thereby also investigating if their exists a difference between simulations and real-life testbed experiments.

The main contributions of this paper are: (a) extending RPL with state-ofthe art link estimators; (b) the analysis on the RPL network performance when using different link estimators in realistic scenario's; (c) a comparison between the results obtained via simulation and via a real-life testbed and; (d) a study on the influence of the underlying Medium Access Control (MAC) protocol and its settings on the performance obtained by the different link estimators.

\section{Related work}

In this section, an overview is given of different routing protocols that rely on link estimation algorithms for making routing decisions. Next to this, different link estimation algorithms are also discussed.

\subsection{Routing protocols for WSNs}

In this work, several link estimation algorithms were implemented into the routing process of RPL. It is a proactive, distance-vector routing protocol specifically designed for WSNs [17]. RPL uses control packets (DIO, DAO and DIS) for building a tree like topology, called a Destination-Oriented Directed Acyclic Graph (DODAG). As RPL routes a packet via the path which minimizes the sum of all link metrics, i.e. the path metric, it takes link quality into account when routing. In contiki RPL [16] only the objective function 0 and Estimated Transmission Count (ETX) methods were available.

Another well-known routing protocol for WSNs is the Collection Tree Protocol (CTP) [8]. CTP also is a pro-active distance-vector routing protocol. It optimizes for data collection applications towards a special node, the data sink. As its name suggests, CTP builds a tree topology with the sink as root using an adaptive beaconing technique. For this it relies on the four-bit (4B) link quality estimator [7] allowing each node to select the best parent.

In the broader field of wireless ad hoc networks, OLSR, BABEL and BATMAN are popular routing protocols [12]. OLSR is a proactive link-state protocol while BABEL and BATMAN are pro-active distance-vector protocols. They all use a variant of the ETX method for estimating the quality of each link.

\subsection{Link estimators}

Link estimation algorithms take the lossy nature and time varying link quality of WSNs into account when determining the best neighbour to forward data to. 
As discussed in [3], link estimators can be classified in hardware- and softwarebased algorithms. The hardware based link estimators use quality indicators (i.e. RSSI and LQI) set by the radio driver after packet reception. Software based link estimators such as Four bit [7] and Fuzzy LQE [4], combine information from multiple network layers into a single metric. There are also very simple link estimators, such as the objective function 0 , that select neighbours based on hop count. In this section a classification of the evaluated link estimators is given.

Hardware based link estimation algorithms Hardware based link estimation algorithms use information provided by the radio (e.g. Received Signal Strength Indicator (RSSI) and Link Quality Indicator (LQI)) to calculate the link metric. Both link layer variables are highly correlated with the PDR [13] and can be used to model link quality. Due to the unstable nature of wireless communication, the raw values can be aggregated with an Exponential Weighted Moving Average (EWMA) filter [3] to improve the estimation. Because LQI and RSSI are only calculated after packet reception, packet-loss is not taken into account. For this reason the link quality of less reliable links can be overestimated.

Software based link estimation algorithms The objective function 0 [15], also referred to as hop count, is a very simple software based link estimator that minimizes the number of hops from the source towards the destination resulting in a route where the preferred parent is the furthest reachable node in the direction of the sink. If the quality of the link with this node is poor, a lot of retransmissions and extra packet loss or energy consumption can occur. On the other hand, in stable networks the overhead is limited for maintaining link information.

More complex software based link estimation algorithms will tackle the aforementioned issue by using historical and/or cross-layer information to make more intelligent decisions when selecting the best links. ETX [11] based algorithms estimate the number of expected transmissions needed to successfully send a packet to each destination by counting the number of attempts needed in previous transmissions. ETX based algorithms select the path with minimal ETX. The Four bit algorithm [7] combines information from multiple OSI-layers to calculate link metrics. It uses LQI and RSSI values to account link quality and combines this with the ETX path metric. Fuzzy logic [4] combines multiple link metrics in a less deterministic way by using membership functions which assign a score in $[0,1]$ for every link metric. These individual scores are combined with an aggregation function such as the Yagger operator. Multiple metrics are aggregated by calculating a weighted mean ( $\beta$ is typical 0.6) of the worst and average value of each metric. This results in a link score which can be inverted to obtain a link metric for routing purposes.

\section{Evaluation Set-up}

The aforementioned link estimation algorithms have been implemented in Contiki RPL [16]. They were evaluated using the Cooja simulator and, experimen- 
tally, on the iMinds w-iLab.t office testbed [1]. Two metrics have been used to determine the performance. First, the PDR is defined as the number of received packets at the RPL sink to the total number of data packets sent in the network. Second, the energy consumption is modelled by the percentage radio-on time which dominates the power usage in sensor nodes.

The network stability was also determined by monitoring the control traffic overhead generated by RPL, e.g. the number of DIO messages and parent switches, and the required MAC retransmissions. These results show a clear correlation with the radio-on time, i.e. unstable networks suffer from a high packet loss and require regular DIO message exchanges and parent switches, resulting in a higher energy consumption. They are therefore not included in this paper.

All simulations and testbed experiments ran twice to determine the variance in results. The experiment and simulation settings can be found in Table 1.

\begin{tabular}{l|l} 
Setting & Value \\
\hline Contiki version & 2.7 \\
MAC protocol & CSMA \\
Radio Duty Cycling & ContikiMAC \\
Maximum number of retransmissions & 5 \\
Neighbor table size & 16 \\
Channel check rate $(\mathrm{Hz})$ & 16 \\
CC2420 transmission power & $-15 \mathrm{dBm}$ \\
Cooja simulation time & 10 minutes \\
w-iLab.t testbed simulation time & 30 minutes \\
Network stabilization time & 1 minute \\
Mobile node speed & $2 \mathrm{~m} / \mathrm{s}(7.2 \mathrm{~km} / \mathrm{h})$
\end{tabular}

Table 1: Experiment and simulation settings

Seven different real-life scenarios were investigated. First, a standard scenario was used where nodes are distributed evenly. The send interval was set to 30 seconds. The second scenario used a sparse topology where nodes are placed further from each-other. The third scenario investigates a dense node topology. The fourth scenario emphasizes the asymmetric behaviour of a real-life sensor node by changing the transmission and interference ranges dependent to the direction. In the fifth scenario, the influence of a bursty send pattern is investigated where each node sends a burst of 3 packets every 90 seconds. The impact of interference was analysed in the sixth scenario. The last scenario introduces mobility by adding two mobile nodes to the standard scenario. In each of the scenario's the sink is placed at the edge of the network to enforce a multi-hop topology.

\subsection{Cooja simulator}

Cooja is a Java-based sensor network simulator for Contiki source code. Since Cooja is a software-based simulator, it doesn't take external interference into account and uses default values for radio driver variables such as LQI and RSSI. The Cooja Unit Disk Graph Medium (UDGM) channel model calculates the 
RSSI value based on the distance between the nodes and uses 37 as default for all LQI values [14]. The UDGM channel model degrades the signal quality based on the distance between nodes. Cooja allows to determine the average radio-on time with the Powertrace plug-in and to simulate mobile nodes with the Mobility plug-in.

Table 2 gives an overview of the different settings used in Cooja to construct the six scenario's.

\begin{tabular}{l|lll} 
& Standard & Mobility & Asymmetric \\
\hline Number of nodes & $8 \times 8=64$ & $8 \times 8+2$ mobile $=66$ & $8 \times 8=64$ \\
Transmission range & $45 \mathrm{~m}$ & $45 \mathrm{~m}$ & Variable \\
Interference range & $60 \mathrm{~m}$ & $60 \mathrm{~m}$ & $60 \mathrm{~m}$ \\
Model of degeneration & UDGM & UDGM & DGRM \\
Send interval & 1 packet/30s 1 packet/30s & 1 packet/30s
\end{tabular}

\begin{tabular}{l|llll} 
& Dense & Sparse & Interference & Bursty \\
\hline Number of nodes & $12 \times 12=1448 \times 8=64$ & $8 \times 8=64$ & $8 \times 8=64$ \\
Transmission range & $45 \mathrm{~m}$ & $30 \mathrm{~m}$ & $45 \mathrm{~m}$ & $45 \mathrm{~m}$ \\
Interference range & $60 \mathrm{~m}$ & $40 \mathrm{~m}$ & $90 \mathrm{~m}$ & $60 \mathrm{~m}$ \\
Model of degeneration & UDGM & UDGM & UDGM & UDGM \\
Send interval & 1 packet/30s 1 packet/30s 1 packet/30s 3 packets/90s
\end{tabular}

Table 2: Specifications of the different scenarios in Cooja.

The standard scenario uses an eight-by-eight grid with a transmission range of 45 meter and an interference range of 60 meter. The default UDGM channel model is used. Each node sends a packet every 30 seconds. The sparse scenario has the same configuration but with a limited transmission $(30 \mathrm{~m})$ and interference $(40 \mathrm{~m})$ range. To simulate a dense network, the standard scenario is extended to a twelve-by-twelve grid.

To investigate link asymmetry, the UDGM model used in the standard scenario is replaced with the Directed Graph Radio Medium (DGRM) channel model which allows to specify different delivery ratios for each directional link in the network. To determine the influence of the send pattern, the default send interval is changed to 90 seconds but now a burst of three packets is transmitted. The impact of interference was analysed by extending the interference range in the standard scenario to 90 meter. The mobility scenario has exactly the same configuration as the standard scenario but with two additional mobile nodes.

\section{2 iMinds w-iLab.t testbed}

Due to limitations of the Cooja simulator, a real-life testbed is needed to reliably evaluate all link estimation algorithms. The iMinds w-iLab.t [1] testbed contains 200 Tmote Sky sensor nodes in a real-life office environment (see figure 1). By varying the amount of nodes in the experiment a standard, sparse and dense networks can be created. Running these tests during daytime introduces extra external interference and varying the transmit power of the Tmote Sky CC2420 
radio [2] allows to increase the asymmetry in the network. The mobile scenario could not be reproduced due to limitations of the iMinds w-iLab.t office testbed. It is important to note that a real-life office testbed always has an inherent

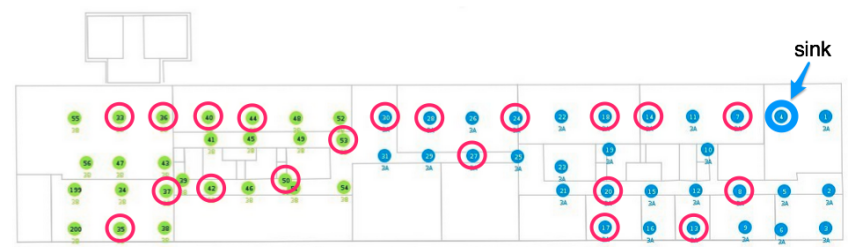

Fig. 1: iMinds w-iLab.t office testbed. Indicated nodes are used in the standard scenario while the sparse scenario only uses 10 nodes and the dense scenario uses all available nodes.

level of asymmetry due to the use of real-life sensor nodes and the occurrence of walls and other obstacles. Next to the asymmetry, an office testbed always has a certain level of external interference from other wireless communication technologies.

\section{Evaluation Results}

\subsection{Cooja simulator}

Figure 2 illustrates the PDR obtained by the different link estimators in each scenario. In the standard network, Four bit and Fuzzy LQE algorithms obtain a $20 \%$ higher packet delivery ratio compared to the other estimators. The PDR in the sparse scenario is nearly the same for all estimators but drops significantly $(12 \%)$ for Four bit and Fuzzy LQE compared to the standard scenario. This is because there are less paths towards the sink allowing less room for optimizations by the more complex algorithms. In dense networks lower PDR ratios are achieved overall, but especially using the RSSI-based and Four bit algorithms (a drop of 50\%). Algorithms based on ETX and Fuzzy LQE perform best in dense networks. The interference scenario only results in a small drop in PDR compared to the standard scenario except for the RSSI and Four bit estimators that show a decrease of $10 \%$. The PDR of all link estimators degrade in the asymmetric scenario and again the Four bit and Fuzzy LQE algorithms suffer the most with a drop of $30 \%$. Now the objective function 0 obtains the best results. The mobility scenario shows very low PDRs overall, especially the RSSI algorithm cannot establish a stable network. Again, the objective function 0 obtains the best results. In the bursty scenario, also a significant drop in PDR can be noted especially for the Four bit algorithm which is very sensitive for changes in the send behaviour, showing a decrease of $30 \%$. Fuzzy LQE performs best in this scenario.

The radio-on time, measured for the different link estimators in each scenario, is shown in Figure 3. The results demonstrate that overall the RSSI algorithm has a much higher energy consumption. This is because the RSSI link estimation 


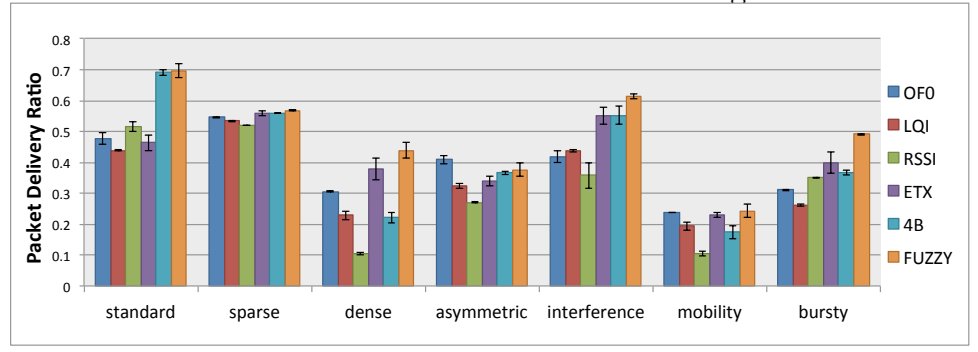

Fig. 2: Packet delivery ratio in the Cooja simulator.

is highly fluctuating, resulting in more RPL parent switches and, consequently, a higher energy consumption. One exception is the sparse scenario where less parent switches are possible. The radio-on time in the sparse and bursty scenarios is nearly identical to the standard scenario. Dense networks on the other hand show an increase in energy consumption, especially for the ETX, Four bit and Fuzzy LQE algorithms. When considering asymmetric links, the results are again nearly identical to the standard scenario except for the Fuzzy LQE which shows a clear increase in radio-on time. In the interference scenario, each estimator consumes slightly more energy, albeit that the differences between them remain constant. In contrast, the scenario including mobility shows a lower energy consumption, but again the differences remain stable.

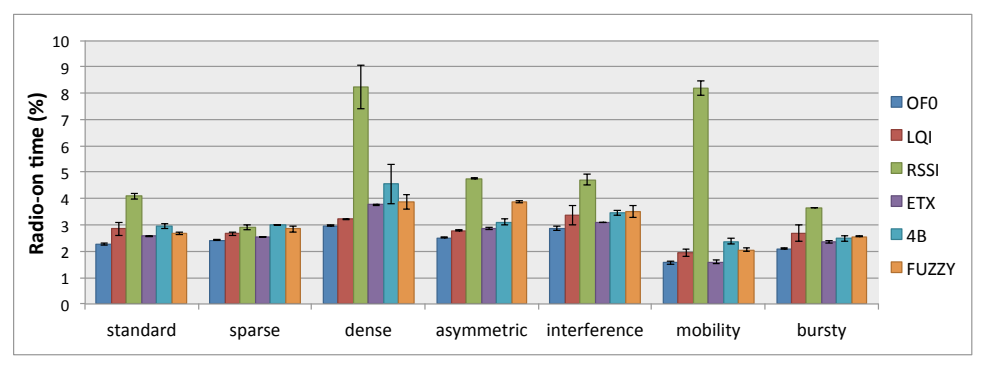

Fig. 3: Average radio-on time (in \%) in the Cooja simulator.

Overall, the simulation results show that combining information from multiple network layers improves the performance of link estimation at the cost of an increased RPL overhead and thus a higher energy consumption. Therefore simple algorithms, such as hop count or LQI, are preferred in sparse or mobile scenarios.

\section{2 iMinds w-iLab.t testbed}

Compared to the simulations, the results show a higher variance in the testbed experiments (see figure 4 and 5). In the standard scenario, the complex algorithms confirm the simulation results, although Fuzzy LQE performs slightly better with less energy consumption. The sparse scenario in the testbed also shows that the objective function 0 obtains a high PDR with a lower radio-on 
time. This is due to a low RPL-overhead and a small amount of retransmissions. In dense networks, the objective function 0, ETX and Fuzzy LQE algorithms obtain the best results. They even show an increase in PDR opposed to the decrease noticed in the simulator. The testbed results confirm that the Four bit algorithm has a degraded performance in dense networks.

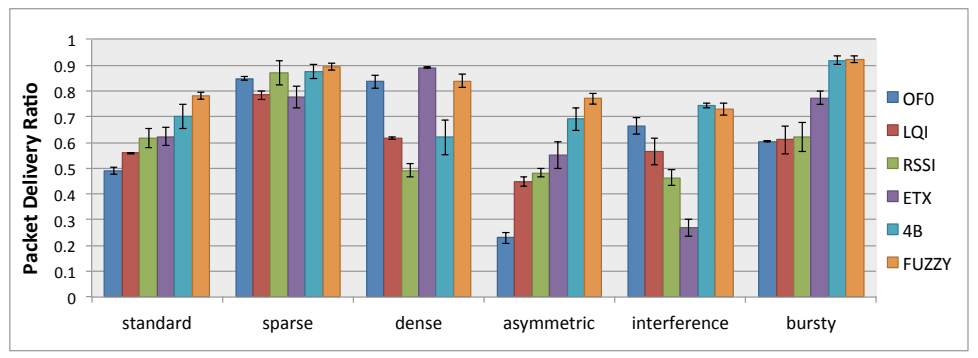

Fig. 4: Packet delivery ratio on the iMinds w-iLab.t testbed.

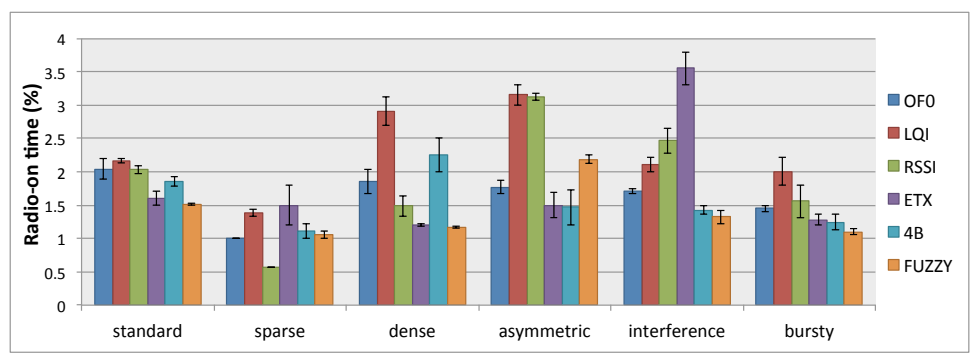

Fig. 5: Average radio-on time (in \%) on the iMinds w-iLab.t testbed.

Compared to the simulation results, the asymmetric scenario shows a decrease in performance for most simple algorithms, but an increase for the complex ones. This can be explained by the network configuration which gives certain nodes higher transmit power to increase asymmetry. The complex algorithms seem to benefit from this higher transmit power. The number of parent switches also decreases, due to larger difference in route qualities less path switches occur. The Four bit algorithm performs a lot better in the interference scenario compared to the simulation results, while ETX performs significantly less. These results clearly show the limitations on the LQI and RSSI values used in Cooja. The bursty scenario shows a performance increase compared to the standard scenario, contradictory to the results in the Cooja simulator.

\section{Influence of RDC/MAC protocol}

Since several link estimators utilize information from received packets, the MAC protocol potentially has a significant influence on the choice of the optimal link estimator. This section investigates the effect of the RDC/MAC protocol and 
its settings on the PDR and radio-on time. In Contiki, the Radio Duty Cycling (RDC) driver is responsible for reducing the energy consumption by turning the radio on and off. The MAC driver is responsible for the CSMA/CA and for packet retransmissions in case of collisions. Both have a major impact on the PDR and radio-on time. ContikiMAC [6] and CSMA are the default RDCand MAC-driver respectively. Other implementations of the RDC driver include X-MAC, CX-MAC and NullRDC. A dummy version of the MAC driver, i.e. nullMac, is also available.

As illustrated in Figure 6 and Figure 7, ContikiMAC outperforms other drivers with a higher PDR and low energy consumption. NullRDC obtains a slightly higher PDR compared to X-MAC, CX-MAC and NullMac, at the cost of a much higher energy consumption because NullRDC never turns the radio off. Despite this, NullRdc has much lower PDR then contikiMAC because it does not include any retransmission. X-MAC and CX-MAC, have less strict timings to support more radio's and require 4 times more energy compared to ContikiMAC. They obtain a PDR close to NullRDC. NullMAC has the lowest energy consumption but also a very low PDR because no retransmission and CCA is performed. All MAC- and RDC-drivers seem to have an equal impact on all link estimation algorithms because the differences in performance between the estimators are retained. Contiki also allows us to specify a Channel Check Rate

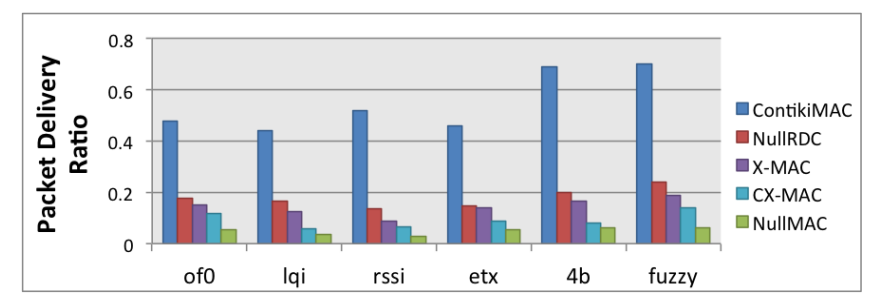

Fig. 6: Packet delivery ratio with several radio duty cycling protocols in the Cooja simulator.

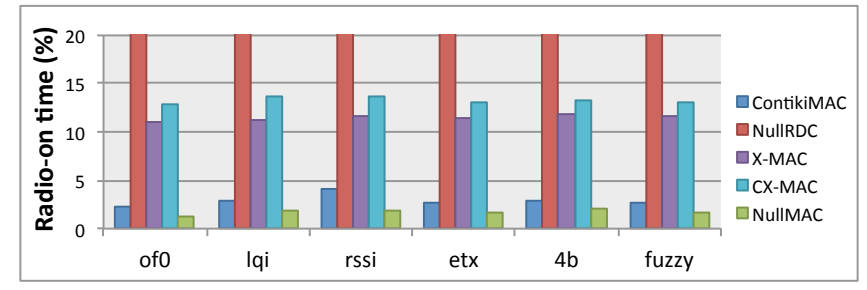

Fig. 7: Radio-on time of several radio duty cycling protocols in the Cooja simulator.

(CCR), which is the frequency the MAC- and RDC-driver use to turn on the radio and check the channel for possible transmissions. A higher CCR results in higher PDR (see figure 8), but requires more energy (see figure 9). Again, varying the CCR seems to has no impact on the type of estimator. The maximum 


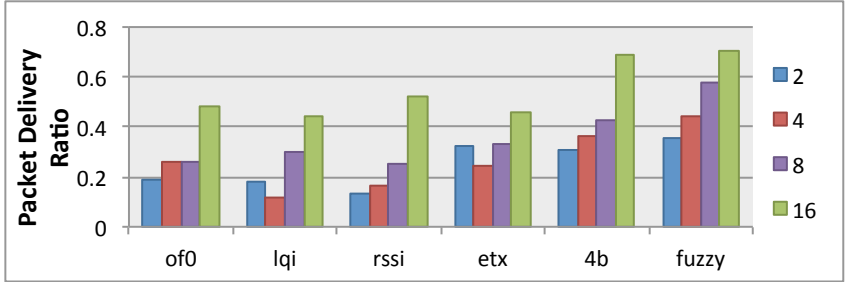

Fig. 8: Packet delivery ratio with several channel check rates in the Cooja simulator.

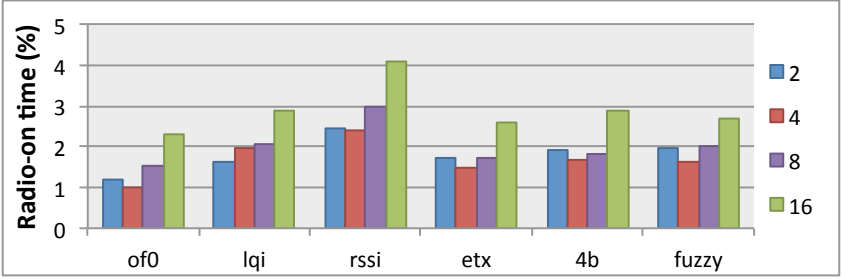

Fig. 9: Radio-on time of several channel check rates in the Cooja simulator.

amount of retransmissions can be freely chosen in ContikiRPL, which allows to limit excessive retransmissions and energy usage. The default value of 5 indicates there will be 6 attempts to send the packet before it gets dropped. Figure 10 indicates how much packets needed multiple retransmissions while performing the standard scenario on the w-iLab.t office testbed.

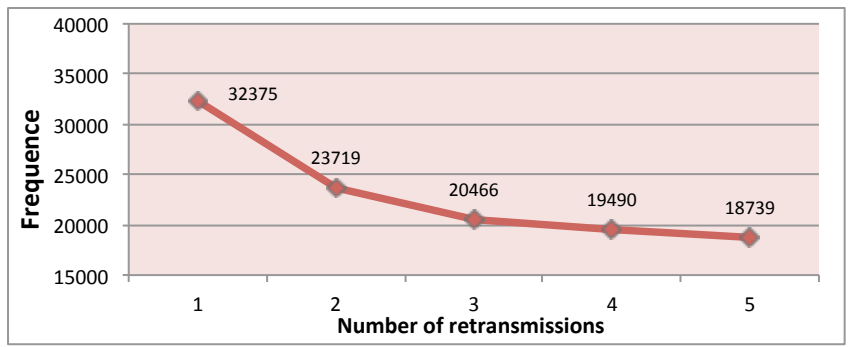

Fig. 10: Influence of maximum number of retransmissions in RPL.

The results show that almost all packets that need two retransmissions, also required three or more retransmissions. This indicates that using more then two or three retransmission only has a minor impact on the packet delivery ratio, but causes a much higher energy consumption.

\section{Conclusions}

Several link estimation algorithms were implemented for ContikiRPL. Next to ContikiRPL's standard objective function 0 and ETX algorithms two additional estimators based on the data link layer RSSI and LQI values and two more complex algorithms, i.e. Four bits and Fuzzy LQE, were added. 
All link estimators were thoroughly evaluated in different dynamic network scenario's both in a simulation environment and in the real-life iMinds w-iLab.t office testbed. After evaluation using PDR and radio-on percentage as performance metrics, it can be concluded that there is no algorithm which performs best in all scenarios. In Table 3 an overview is given of the best performing algorithms for each scenario. Fuzzy LQE is the best choice in most scenario's while the Objective function 0 is better suited for the sparse and mobile scenarios. ETX is also a good choice in the dense and mobility scenario. The complex Four bit algorithm is only better in the asymmetric scenario at the cost of a higher energy consumption.

\begin{tabular}{l|l} 
Scenario & Best link estimation algorithm \\
\hline Standard & Fuzzy LQE \\
Sparse & Objective function 0 \\
Dense & Fuzzy LQE or ETX \\
Asymmetric & Four bit or Fuzzy LQE \\
Interference & Fuzzy LQE \\
Bursty & Fuzzy LQE \\
Mobility & ETX or Objective function 0
\end{tabular}

Table 3: Overview of the conclusions of this research.

The results obtained in the simulation environment show a high discrepancy with the results obtained in the iMinds w-iLab.t testbed. We can hence conclude that simulators are decent tools for algorithm development but lack capabilities to model real-life network characteristics and deliver reliable results.

The impact of the choice of RDC- and MAC-protocol was also investigated. The results show that there is no influence on the differences in performance between multiple link estimation algorithms but there is a difference in the overall performance and energy consumption of the network. The CCR also has a major impact on the PDR and energy consumption. It was also shown that there should only be two retransmission because more retransmissions only has a minor effect on the PDR at the cost of a much higher energy consumption.

Future work could investigate the influence of the transmit power, node distance or modulation scheme on the quality of a link. Moreover, since real-life networks are often a combination of multiple scenarios it can be interesting to combine different link estimation algorithms in a single network. The link estimators can also be further optimized. For instance, the mapping of LQI and RSSI values to a link metric can be varied, the thresholds in the Four bit algorithm can be changed and different membership and aggregation functions can be used in the Fuzzy LQE algorithm. An optimal link estimation algorithm would be self-adapting, changing the link estimator algorithm (settings) based on the observed network characteristics.

\section{Acknowledgments}

This work was partially supported by the Fund for Scientific Research-Flanders (FWO-V), project "ASN: wireless Acoustic Sensor Networks", grant \#G.0763.12, 
and the agency for Innovation by Science and Technology Flanders (IWT-V), project "SAMURAI: Software Architecture and Modules for Unified RAdIo control", and the iMinds IoT Strategic Research program, and European Commission Horizon 2020 Programme under grant agreement n645274 (WiSHFUL).

\section{References}

1. iMinds w-iLab.t website. http://ilabt.iminds.be/iminds-wilabt-overview, online, last visited on July 12, 2015

2. Texas Instruments CC2420 Radio Datasheet. http://www.ti.com/lit/ds/ symlink/cc2420.pdf, online, last visited on July 12, 2015

3. Baccour, N., Koubâa, A., Mottola, L., Zúñiga, M.A., Youssef, H., Boano, C.A., Alves, M.: Radio link quality estimation in wireless sensor networks: A survey. ACM Trans. Sen. Netw. 8(4), 34:1-34:33 (Sep 2012)

4. Baccour, N., Koubâa, A., Youssef, H., Jamâa, M.B., Do Rosario, D., Alves, M., Becker, L.B.: F-lqe: A fuzzy link quality estimator for wireless sensor networks. In: Wireless Sensor Networks, pp. 240-255. Springer (2010)

5. Dawans, S., Duquennoy, S., Bonaventure, O.: On link estimation in dense rpl deployments. In: Local Computer Networks Workshops (LCN Workshops), 2012 IEEE 37th Conference on. pp. 952-955 (Oct 2012)

6. Dunkels, A.: The contikimac radio duty cycling protocol. Tech. rep., SICS (2011)

7. Fonseca, R., Gnawali, O., Jamieson, K., Philip, L.: Four bit wireless link estimation. In: Proceedings of the Sixth Workshop on Hot Topics in Networks (HotNets (2007)

8. Gnawali, O., Fonseca, R., Jamieson, K., Kazandjieva, M., Moss, D., Levis, P.: Ctp: An efficient, robust, and reliable collection tree protocol for wireless sensor networks. ACM Trans. Sen. Netw. 10(1), 16:1-16:49 (Dec 2013)

9. Gungor, V.C., Korkmaz, M.K.: Wireless Link-Quality Estimation in Smart Grid Environments. International Journal of Distributed Sensor Networks (2012)

10. Iova, O., Theoleyre, F., Noel, T.: Stability and efficiency of rpl under realistic conditions in wireless sensor networks. In: Personal Indoor and Mobile Radio Communications (PIMRC), 2013 IEEE 24th International Symposium on (2013)

11. Javaid, N., Javaid, A., Khan, I., Djouani, K.: Performance study of etx based wireless routing metrics. In: Computer, Control and Communication (IC4) (2009)

12. Klein, A., Braun, L., Oehlmann, F.: Performance study of the better approach to mobile adhoc networking (batman) protocol in the context of asymmetric links. In: World of Wireless, Mobile and Multimedia Networks (WoWMoM) (2012)

13. Meier, A., Rein, T., Beutel, J., Thiele, L.: Coping with unreliable channels: Efficient link estimation for low-power wireless sensor networks. In: Networked Sensing Systems, 2008. INSS 2008. 5th International Conference on. pp. 19-26 (June 2008)

14. Osterlind, F., Dunkels, A., Eriksson, J., Finne, N., Voigt, T.: Cross-Level Sensor Network Simulation with COOJA. In: Local Computer Networks (LCN) (2006)

15. Thubert, P.: Objective Function Zero for the Routing Protocol for Low-Power and Lossy Networks (RPL). RFC 6552 (Proposed Standard) (Mar 2012)

16. Tsiftes, N., Eriksson, J., Dunkels, A.: Low-power wireless ipv6 routing with contikirpl. In: International Conference on Information Processing in Sensor Networks. IPSN '10, ACM (2010)

17. Winter, T., Thubert, P., Brandt, A., Hui, J., Kelsey, R., Levis, P., Pister, K., Struik, R., Vasseur, J., Alexander, R.: RPL: IPv6 Routing Protocol for Low-Power and Lossy Networks. RFC 6550 (Proposed Standard) (Mar 2012) 